\title{
Fourier Analysis of Slow and Fast Image Propagation through Single and Coupled Image Resonators
}

\author{
Parvin Sultana, ${ }^{1}$ Takahiro Matsumoto, ${ }^{2}$ and Makoto Tomita ${ }^{1,3}$ \\ ${ }^{1}$ Graduate School of Science and Technology, Shizuoka University, 836 Ohya, Suruga-ku, Shizuoka 422-8529, Japan \\ ${ }^{2}$ Research and Development Center, Stanley Electric Corporation, 5-9-5 Tokodai, Tsukuba 300-2635, Japan \\ ${ }^{3}$ Department of Physics, Faculty of Science, Shizuoka University, 836 Ohya, Suruga-ku, Shizuoka 422-8529, Japan
}

Correspondence should be addressed to Parvin Sultana, psmony@gmail.com

Received 30 July 2012; Accepted 12 October 2012

Academic Editor: Sulaiman Wadi Harun

Copyright (C) 2012 Parvin Sultana et al. This is an open access article distributed under the Creative Commons Attribution License, which permits unrestricted use, distribution, and reproduction in any medium, provided the original work is properly cited.

\begin{abstract}
We applied Fourier space analysis to a comprehensive study of the propagation of pulsed two-dimensional images through single and coupled image resonators. The Fourier method shows that the image can propagate through the resonator successfully as long as the spatial and temporal Fourier components of the image are within the bandwidth of the amplitude and phase transfer functions. The relevant steep dispersion of the cavity can yield delayed or advanced images. The Fourier method reproduces characteristic aspects of the experimental observations of the image propagation, and also predicts new aspects, such as the spatial image profile dependence on the observation time and the coupling strength. To demonstrate the time evolution of the experiment, space- and time-resolved image propagations were performed using a streak camera.
\end{abstract}

\section{Introduction}

When propagating through media, the velocity of an optical wave packet depends on the variation of the refractive index. In recent years, there has been increasing interest in media in which the refractive index changes drastically with respect to changes in frequency, resulting in significant modification in the corresponding group velocities of the optical pulses. We refer to this phenomenon as "slow light" or "fast light." Experimental demonstrations of slow and fast light have been realized based on stimulated Brillouin scattering in optical fibers [1], electromagnetically induced absorption (EIA) and transparency (EIT) in atomic vapors [2-5], structural dispersion in microsphere-optical fiber systems [6], coupled-resonator-induced transparency (CRIT) with classical light [7], and squeezed vacuum studies [8]. Recently, various groups have reported stopping light altogether, which leads to new methods of storing information $[9,10]$.

It is challenging to apply slow and fast light techniques to two-dimensional (2D) image propagation. Alloptical methods for delaying images have great potential in many applications, including holography and optical pattern correlation measurements or futuristic all-optical image routers for large capacity image information. The first experimental demonstration of an all-optical image delay was reported in Cs atoms [11]. The storage and retrieval of a transverse image in an atomic vapor was demonstrated using a technique based on EIT [12, 13]. Recently, researchers succeeded in eliminating arbitrary paraxial image diffraction by manipulating the susceptibility in momentum space [14]. This work opens up new possibilities for utilizing EIT to realize high-resolution imaging and position-independent beam expansion.

We reported an optical image propagation experiment using single and coupled image resonators. Two-dimensional images encoded on optical pulses were either advanced or delayed through a single image resonator using relevant dispersion [15]. Further, 2D tunable delayed images were demonstrated experimentally using CRIT. The coupled resonator is recognized as the all-optical analogue of EIT [16]. A steep positive slope induced by the transparency effect of the second cavity, in otherwise opaque absorption from the first cavity, allows the observation of the slow image. Because the image resonator was based on structural dispersion, it had many advantages compared with atomic systems. In addition to the relative design simplicity, the 


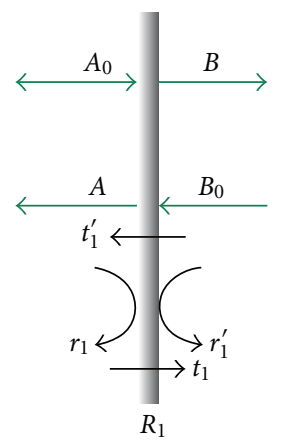

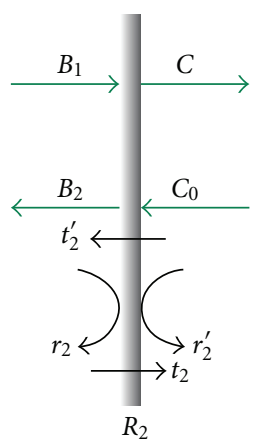

(a)
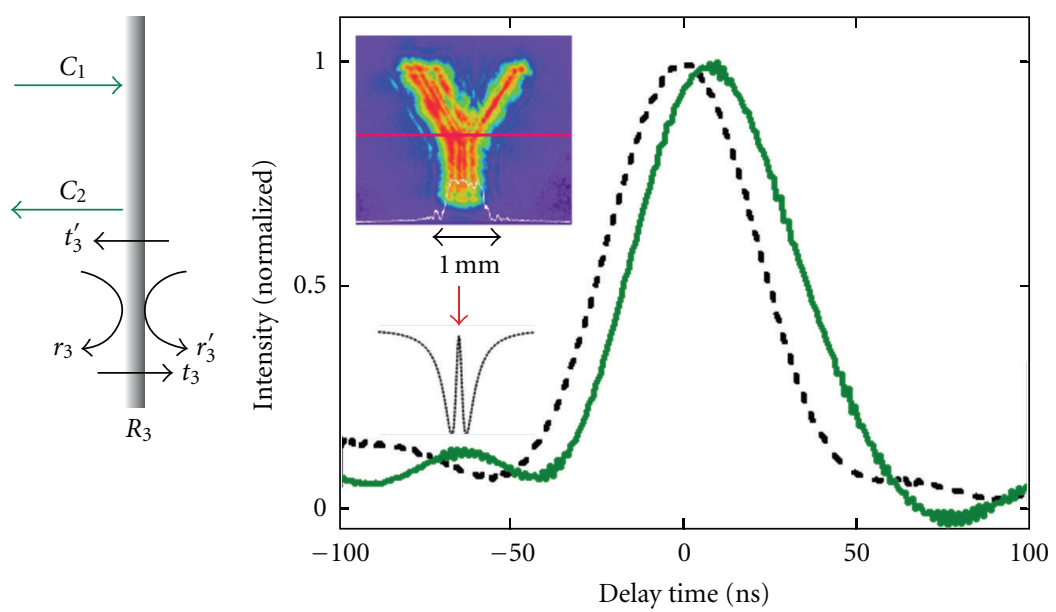

(b)

FIGURE 1: (a) Schematic illustration of the reflected and transmitted image propagation through a coupled Fabry-Perot image resonator. In this figure, $R_{1}, R_{2}$, and $R_{3}$ are the reflectivities of the three mirrors that comprise the coupled cavity. (b) An example of delayed pulsed $2 \mathrm{D}$ image propagation through the coupled-resonator-induced transparency. The insets show the reflected image (top) at the center of the transparency window (CRIT), indicated by the red arrow in the frequency spectrum (bottom).

advantages of the photonic structures are that the available frequencies are not restricted to intrinsic atomic transitions; an additional laser beam is not required to prepare the slow light medium; the system is not affected by Doppler motion. Although there have been studies demonstrating pulsed 2D image propagation, until now there has been no comprehensive theoretical analysis for image propagation through the single and coupled resonators, specifically from the dispersion point of view. In contrast to real-space analysis $[15,16]$, in this study we applied Fourier space analysis to study the propagation of $2 \mathrm{D}$ images encoded on temporal pulses through single and coupled image resonators, comprised of Fabry-Perot resonators. Real-space analysis provided an intuitive interpretation; however, this approach is not easily applied to complicated images or coupled resonators. The Fourier method reproduces the characteristic aspects of the experimental observations on the image propagation reported to date and also predicts the time evolution of image propagation through the cavity.

Fabry-Perot resonators are widely used experimentally and often discussed as a model cavity. The longitudinal domain effects on frequency filtering, the laser cavity, the phase-amplitude converter, and nonlinear characteristics have been studied extensively. However, little is known on the general transverse effect of a cavity on an image, particularly from a dispersion point of view. In this study, a planar cavity is treated as a parallel processing system with image transmission. Rays making a small angle with respect to the planar cavity axis escape from the cavity after a great number of reflections. However, as long as the spatial and temporal Fourier components of the image are within the bandwidth of the amplitude and phase transfer functions, the images can successfully propagate through the cavity. It has been discussed that particular types of cavities, such as confocal or hemiconfocal cavities with transverse degenerate cavity modes, could transmit a portion of the image [17, 18]; this provides interesting possibilities regarding the future of image resonators.

\section{Fourier Analysis for Image Propagation}

We applied Fourier space analysis to the wave vector $(k)$ frequency $(v)$ domain. Figure 1(a) shows a schematic of a coupled Fabry-Perot cavity consisting of three plane parallel mirrors with reflectivity $R_{i}=r_{i} r_{i}^{*}=r_{i}^{\prime} r_{i}^{\prime *}$ and transitivity $T_{i}=t_{i} t_{i}^{*}=t_{i}^{\prime} t_{i}^{\prime *}$, where $i=1,2$, and 3 correspond to the first, second, and third mirrors, respectively. This configuration yielded the coupled-resonator-induced transparency. The single cavity configuration can also be treated by setting $R_{3}=0$. Figure $1(\mathrm{~b})$ is an example of delayed pulsed $2 \mathrm{D}$ image propagation through the coupled-resonator-induced transparency. The insets (top) show the reflected image at the center of the transparency window (CRIT), indicated by the red arrow in the frequency spectrum (bottom). The pulse peak was delayed by $10.6 \mathrm{~ns}$, due to the steep dispersion of the resonator. The incident electric field of the image with wave vectors $k_{x}, k_{y}$ and frequency $v$ is denoted as $A_{0}\left(k_{x}, k_{y}, v\right)$; the output electric field is given as $A\left(k_{x}, k_{y}, \nu\right)$. The notation $B\left(k_{x}, k_{y}, v\right), B_{0}\left(k_{x}, k_{y}, v\right), B_{1}\left(k_{x}, k_{y}, v\right)$, and $B_{2}\left(k_{x}, k_{y}, v\right)$ refers to the electric fields in the first cavity; $C\left(k_{x}, k_{y}, \nu\right)$, $C_{0}\left(k_{x}, k_{y}, v\right), C_{1}\left(k_{x}, k_{y}, v\right)$, and $C_{2}\left(k_{x}, k_{y}, v\right)$ correspond to the electric fields in the second cavity, as shown in Figure 1. The electric fields of the cavities are given by

$$
\begin{aligned}
& A=A_{0} r_{1}+B_{0} t_{1}^{\prime}, \\
& B=A_{0} t_{1}+B_{0} r_{1}^{\prime}, \\
& B_{2}=B_{1} r_{2}+C_{0} t_{2}^{\prime},
\end{aligned}
$$




$$
\begin{gathered}
C=B_{1} t_{2}+C_{0} r_{2}^{\prime}, \\
C_{2}=C_{1} r_{3},
\end{gathered}
$$

where

$$
\begin{gathered}
B_{1}=B \exp \left[-\rho_{1}+i \phi_{1}\right], \\
B_{0}=B_{2} \exp \left[-\rho_{1}+i \phi_{1}\right], \\
C_{1}=C \exp \left[-\rho_{2}+i \phi_{2}\right], \\
C_{0}=C r_{3} \exp \left[-2 \rho_{2}+2 i \phi_{2}\right] .
\end{gathered}
$$

Here, $\rho_{1}, \rho_{2}$ are the losses inside the cavity. The phase relation for the two cavities can be written as $\phi_{j}\left(k_{x}, k_{y}, v\right)=i L_{j}\left(k_{0}^{2}-\right.$ $\left.k_{x}^{2}-k_{y}^{2}\right)^{1 / 2}$, where $i$ is the imaginary number, $j=1,2$, and $k_{0}=2 \pi v / c$, where $c$ is the velocity of light, and $v$ is the frequency of the laser. One part of the incident light does not enter the medium, but instead is reflected from the boundary of the first mirror surface and appears directly at the output of the system; we refer to this part as the direct component. The other part of the incident light enters the cavities, where it is reflected multiple times before exiting through the first mirror as a multiple-reflected component. The total electric field at the output of this system is represented as the sum of the direct- and multiple-reflected components. Therefore, the resultant reflected image is given by

$$
\begin{aligned}
T\left(k_{x}, k_{y}, v\right) & =\left\{r_{1}-\frac{t_{1} t_{1}^{\prime} T_{2} e^{-2 \rho_{1}+2 i \phi_{1}}}{1-r_{1}^{\prime} T_{2} e^{-2 \rho_{1}+2 i \phi_{1}}}\right\} \\
& =\left|T\left(k_{x}, k_{y}, v\right)\right| e^{i \theta\left(k_{x}, k_{y}, \nu\right)},
\end{aligned}
$$

where

$$
T_{2}\left(k_{x}, k_{y}, v\right)=r_{2}-\frac{t_{2} t_{2}^{\prime} r_{3} e^{-2 \rho_{2}+2 i \phi_{2}}}{1-r_{2}^{\prime} r_{3} e^{-2 \rho_{2}+2 i \phi_{2}}} .
$$

Here, $T\left(k_{x}, k_{y}, v\right)$ is the amplitude transfer function of the coupled image resonator. The first term in (3) is the direct-reflected component, and the second term is the sum of the multiple-reflected components. For the single image resonator, the third mirror was removed (i.e., $R_{3}=$ $0)$. Hence, multiple components reflected back and forth between the first and second mirrors that made up the single cavity.

Provided that the system is linear, the transfer function of (3) operates on the input image to produce an output image. We can write the Fourier transformation of the real space image encoded on optical pulses $A_{0}(x, y, \tau)$ as

$$
A_{0}\left(k_{x}, k_{y}, \nu\right)=\iiint_{-\infty}^{\infty} A_{0}(x, y, \tau) e^{i\left(k_{x} x+k_{y} y\right)} e^{-i 2 \pi \tau v} d x d y d \tau .
$$

By transforming $A_{0}(x, y, \tau)$, we are basically decomposing the input image into the complex exponential functions of the various spatial and temporal frequencies $\left(k_{x}, k_{y}, v\right)$. Multiplication of the input spectrum by the transfer function then takes into account the effects of the system on each Fourier component. As a result, when an image propagates through the cavity, the amplitude and phase of each Fourier component will be modified as follows:

$$
A\left(k_{x}, k_{y}, \nu\right)=A_{0}\left(k_{x}, k_{y}, \nu\right) T\left(k_{x}, k_{y}, \nu\right)
$$

Finally, taking the inverse Fourier transform of the output spectrum produces the real output image [19]:

$$
A(x, y, \tau)=\iiint_{-\infty}^{\infty} A\left(k_{x}, k_{y}, v\right) e^{-i\left(k_{x} x+k_{y} y\right)} e^{i 2 \pi \tau v} d k_{x} d k_{y} d \nu .
$$

We calculated the image propagation through the single and coupled image resonators using (5)-(7). A variety of configurations can be considered for both single and coupled image resonators, depending on the coupling conditions of the cavities. Both slow and fast images come from the steep slope of the dispersion. In the reflection geometry, we obtained both anomalous and normal dispersion that depended on $R_{1}$ and $R_{2}$. When $R_{1}>R_{2}$, the coupling is weak compared with the loss; this is known as the under coupling condition. Under this condition, a negative gradient $\partial \theta / \partial v<0$ (1) appears in the dispersion curve within the resonance dip. Such a slope results in anomalous dispersion, and a fast image is expected. When $R_{1}<R_{2}$, the coupling is strong compared with the loss; this is referred to as the over coupling condition. In this case, the phase increases monotonically as the frequency of the laser light is increased, that is, $\partial \theta / \partial v>0$, and slow light is expected. When $R_{1}=R_{2}$, the coupling and the loss become equal, and the reflection minimum becomes zero. This is known as the critical coupling condition.

Figures 2(a)-2(c) show the intensity transfer function for single and coupled resonators in $k$-space and $\nu$-space $|T(k, v)|^{2}$. The $k$-space cross-section of the intensity transfer function, that is, $|T(k, 0)|^{2}$, is shown along the vertical axis. Figures $2(\mathrm{~d})-2(\mathrm{f})$ are the $\nu$-space cross-sectional profiles, $|T(0, v)|^{2}$, and Figures $2(\mathrm{~g})-2(\mathrm{i})$ are the reflected phase shift as a function of the detuning frequency. To discuss the image's optical resolution limitations, we defined the cavity bandwidths of the transfer function $\left|T\left(k_{x}, k_{y}, v\right)\right|^{2}$ in $k$ and $\nu$ space as $\Delta k$ and $\Delta v$, respectively. The Fourier components of the image outside the bandwidth were lost or phase-shifted, and the final images showed deterioration. Figure 2(b) shows the intensity transfer functions for the coupled resonator at $\Delta v_{12}=v_{2}-v_{1}=0$, that is, when two cavities are adjusted to be on-resonant by tuning the cavity lengths. Here, $v_{1}$ and $v_{2}$ are the resonance frequencies of the first and second cavities, respectively. When $\Delta v_{12}=0$, a narrow transmission peak appeared at $\delta v=v-v_{2}=0$ due to the destructive interference between the two resonators, exhibiting CRIT [16]. Consider the situation in which the two cavities have different resonance frequencies, that is, $\Delta \nu_{12} \neq 0$, as shown in Figure 2(c). For $\Delta \nu_{12} \neq 0$, a sharp asymmetric structure appeared, due to the Fano effect. For $\Delta \nu_{12}>0$, this asymmetry increased the relevant $k$-space bandwidth; this may be useful for fine image propagation. 


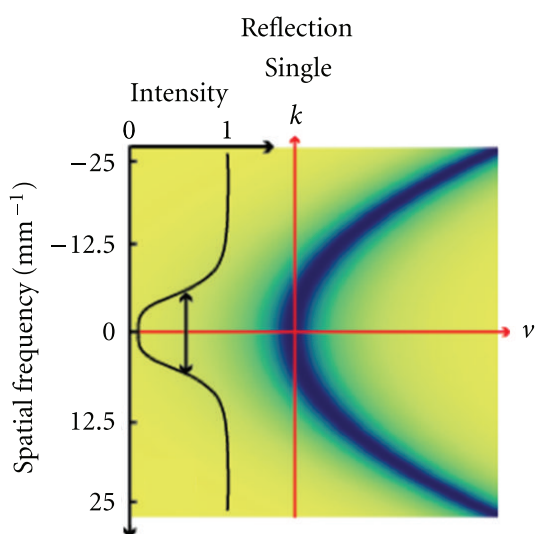

(a)

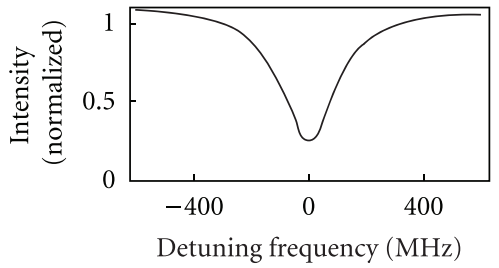

(d)

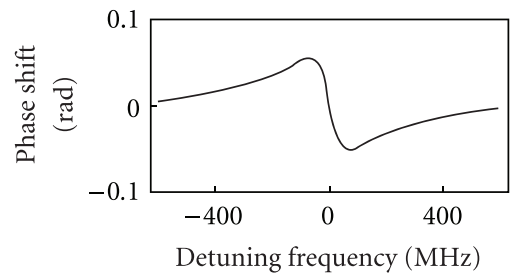

(g)
Reflection

Coupled $\left(\Delta v_{12}=0\right)$

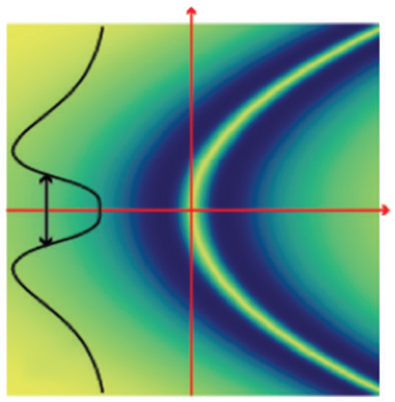

(b)

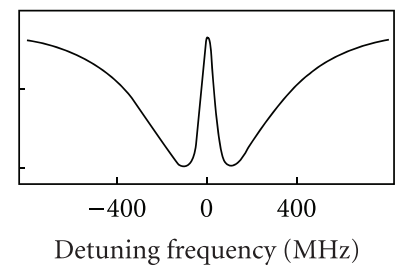

(e)

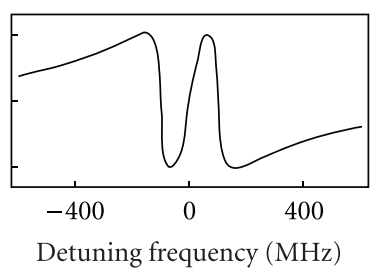

(h)
Reflection

Coupled $\left(\Delta v_{12} \neq 0\right)$

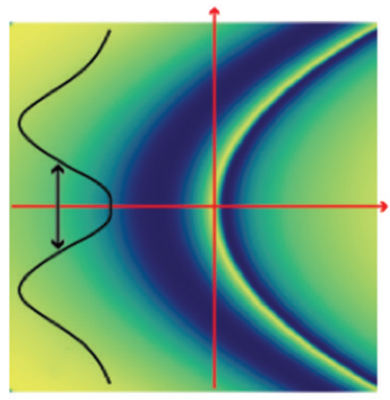

(c)

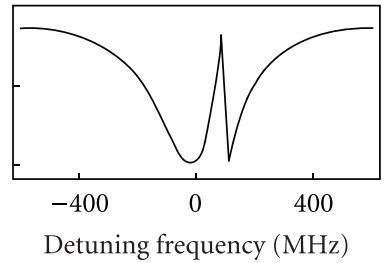

(f)

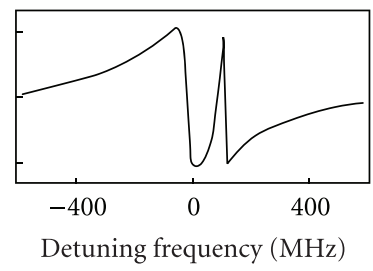

(i)

FIGURE 2: (a)-(c) Calculated intensity transfer functions $|T(k, v)|^{2}$ for (a) a single image resonator, (b) coupled image resonator with $\Delta \nu_{12}=0$, and (c) coupled image resonator with $\Delta \nu_{12} \neq 0$. The black solid curves along the $k$-axis in Figures (a)-(c) are the $k$-space crosssections $|T(k, 0)|^{2}$. (d)-(f) $\nu$-space cross-sections $|T(0, v)|^{2}$. (g)-(i) Calculated phase shift as a function of the detuning frequency. Note that, in (a), the reflectances of the mirrors were $R_{1}=0.9$ and $R_{2}=0.7$, and the cavity length was $L_{1}=10 \mathrm{~cm}$; in (b) and (c), the reflectances were $R_{1}=0.5, R_{2}=0.9$, and $R_{3}=0.99$, and the cavity lengths were $L_{1}=5 \mathrm{~cm}$ and $L_{2}=10 \mathrm{~cm}$, respectively. The $k$-space cavity bandwidths in (a), (b), and (c) were $\Delta k=10.1 \mathrm{~mm}^{-1}, \Delta k=9.8 \mathrm{~mm}^{-1}$, and $\Delta k=13.3 \mathrm{~mm}^{-1}$, respectively, as indicated by the solid black double-arrow lines.

Using the parameters from Figures $2(\mathrm{~b})$ and $2(\mathrm{c})$, the $k$-space bandwidth $(\Delta k)$ can be increased from $\Delta k=9.8 \mathrm{~mm}^{-1}$ to $\Delta k=13.3 \mathrm{~mm}^{-1}$. It is depicted in Figures $2(\mathrm{~b})$ and $2(\mathrm{c})$ by the black double-arrow line. The $k$-space bandwidth for the single resonator in Figure 2(a) is $\Delta k=10.1 \mathrm{~mm}^{-1}$.

\section{Stationary Image Propagation through Single and Coupled Resonators}

Using the transfer function shown in Figure 2, we first examined the propagation of $2 \mathrm{D}$ images $E(x, y)$ through single and coupled image resonators. We used a continuous wave input beam for the transmission of a monochromatic image in the $x-y$ domain. Figure $3(\mathrm{a})$ is the original image injected into the cavity. Figure $3(\mathrm{~b})$ is the Fouriertransformed input image. The full width at half maximum of the Fourier image was $\delta k=4.9 \mathrm{~mm}^{-1}$. Figures $3(\mathrm{c})-3(\mathrm{e})$ are the reflected images obtained from the single resonator using a continuous wave input beam for the under, critical, and over coupling conditions, respectively. Several characteristic aspects of the $2 \mathrm{D}$ image propagation that was obtained experimentally $[15,16]$ were also reproduced in the Fourier analysis. We observed that the edge region of the image was enhanced for the under coupling condition (Figure 3(c)). This edge-enhanced effect was most prominent for the critical coupling condition (Figure 3(d)), in which we observed edge filtering. For the over coupling condition, the hollow effect was observed, that is, a superimposed outline structure appeared around the original image (Figure 3(e)). Figures $3(\mathrm{f})$ and $3(\mathrm{~g})$ are the output reflected images observed from the coupled image resonator $\left(R_{1}=0.5, R_{2}=0.9, R_{3}=\right.$ 0.99 , and $L_{1}=5 \mathrm{~cm}, L_{2}=10 \mathrm{~cm}, \Delta k=9.0 \mathrm{~mm}^{-1}$, $\Delta \nu=80.6 \mathrm{MHz})$ using a continuous wave input beam and temporal pulses of $25 \mathrm{~ns}(\delta \nu=17.6 \mathrm{MHz})$, respectively. It is clear from Figures $3(\mathrm{f})$ and $3(\mathrm{~g})$ that the pulsed image can propagate through the image cavity at almost the same quality as the steady-state image, provided that the spatial 


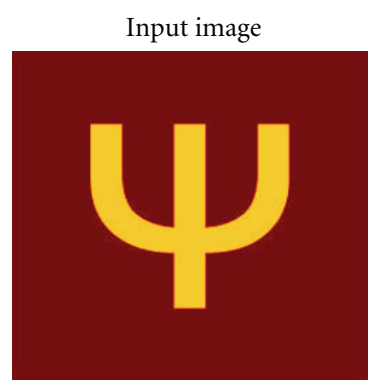

(a)

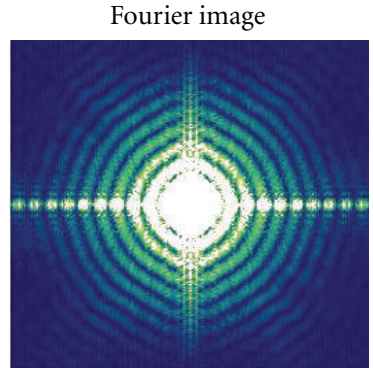

(b)

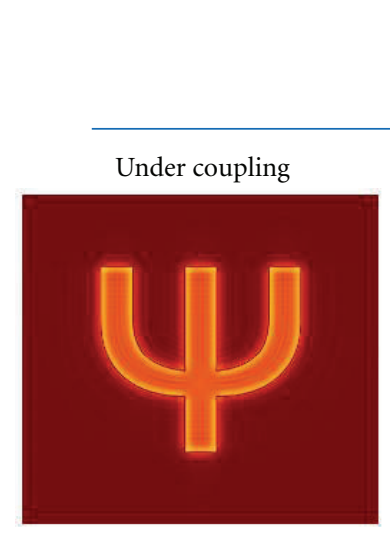

(c)

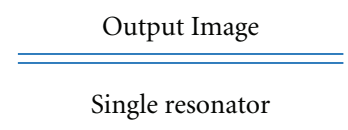

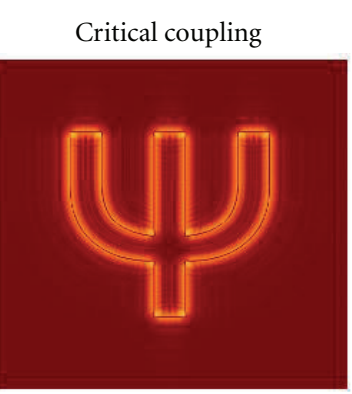

(d)

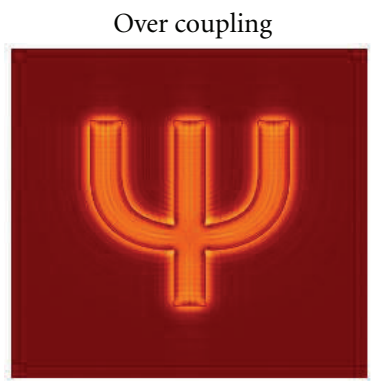

(e)

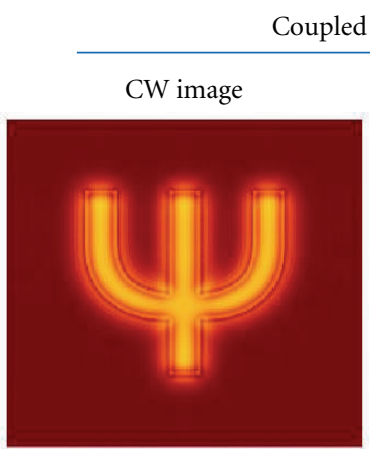

(f)

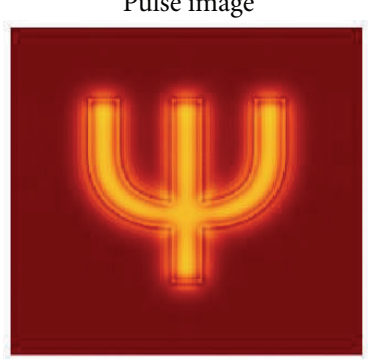

(g)

Figure 3: (a) Original image injected into the cavity. (b) Fourier-transformed input image. The full width at half maximum of the Fourier image is $\delta k=4.9 \mathrm{~mm}^{-1}$. (c)-(e) Reflected images obtained using continuous wave input light for under $\left(R_{1}=0.7, R_{2}=0.5, \Delta k=\right.$ $\left.13.0 \mathrm{~mm}^{-1}\right)$, critical $\left(R_{1}=0.5, R_{2}=0.5, \Delta k=9.0 \mathrm{~mm}^{-1}\right)$, and over coupling $\left(R_{1}=0.5, R_{2}=0.7, \Delta k=13.0 \mathrm{~mm}^{-1}\right)$ conditions, respectively. (f) and (g) Reflected output images from the coupled image resonator $\left(R_{1}=0.5, R_{2}=0.9, R_{3}=0.99, L_{1}=5 \mathrm{~cm}, L_{2}=10 \mathrm{~cm}, \Delta k=9.0 \mathrm{~mm}{ }^{-1}\right.$, $\Delta v=80.6 \mathrm{MHz})$ using a continuous wave input beam and temporal pulses of $25 \mathrm{~ns}(\delta \nu=17.6 \mathrm{MHz})$, respectively.

and temporal Fourier components of the images are within the bandwidth of the cavity.

It is convenient to separate the direct- and multiplereflected components to interpret the spatial and temporal characteristics of the propagated image. The total output image is the sum of the direct component and the $\pi(\mathrm{rad})$ phase-shifted and time-delayed multiple-reflected components [15]. Because the first mirror reflects all of the $k$-space Fourier components, the $k$-space bandwidth is effectively infinite for the direct component. On the other hand, for multiple components, the bandwidth is restricted by the cavity resonance mode; hence, the higher order components are lost. As a consequence of the limited bandwidth, the edge region of the resultant multiple-reflected components is broadened. This was apparent in the single resonator system, as shown in Figures 3(c)-3(e). Multiple-reflected components were the major components for the over coupling condition $\left(R_{1}<R_{2}\right)$; hence, the spatially broadened multiple component formed the hollow structure around the original image (Figure 3(e)). In contrast, for the under coupling condition $\left(R_{1}>R_{2}\right)$, the direct component played the major role. As a result, the broadening effect on the edge region of the multiple components was weak. The destructive interference between the direct and multiple components in the edge region was not perfect, resulting in enhancement of the edge structure (Figure 3(c)). This effect was most evident under the critical coupling condition (Figure 3(d)). 


\section{Time Evolution of the Pulsed Image Propagation}

We next considered the time evolution of the $2 \mathrm{D}$ image encoded on the pulses, $E(x, y, t)$, using the transfer function shown in Figure 2. Figure 4(f) illustrates the cross-section that was considered for time evolution analysis. Although we were mostly interested in the time evolution of $2 \mathrm{D}$ image propagation, it was convenient to show certain crosssections of the image over the course of the measurement, to observe the other sections of the image, which behaved in a similar manner. Figures $4(a)-4(d)$ show the Fourier analysis for the time evolution of the cross-section in reflected and transmitted images in the space-time $(x-t)$ domain, using a single resonator. Figure 4(e) shows the input image of width $1.4 \mathrm{~mm}$ (the horizontal axis) encoded on the temporal Gaussian pulse of $25 \mathrm{~ns}$ (the vertical axis). Figures 4(a), $4(\mathrm{~b})$, and 4(c) show the reflected images from the single resonator for under, critical, and over coupling conditions, respectively. The vertical profiles on the left side of Figures 4(a)-4(d) show the temporal profiles of the input and output images at the center position, indicated by the white and yellow curves, respectively. For the under coupling condition (Figure 4(a)), the temporal profile of the output image was advanced compared with the input image, thus, showing a manifestation of the fast image. Figure 4(c) shows the time evolution calculated for the over coupling condition. The hollow structures around the original image consisted of diffracted multiple components only, simply delayed by the effective optical path length. A similar effect was also shown in Figure 3(e). The center of the cross-section was more delayed as a result of the destructive interference between the direct and multiple components, which is a manifestation of the slow image. Under the critical coupling condition (Figure 4(b)), the temporal profile of the output image was split as a result of the destructive interference effect of the direct- and multiple-reflected components.

In Figures $4(\mathrm{a})-4(\mathrm{~d})$, the spatial profiles at earlier, intermediate, and later times are plotted on the right hand side, indicated by the notations 1,2 , and 3 , respectively. It is interesting to note that the time-resolved spatial profiles transformed continuously from under to critical to over coupling conditions. The spatial profile at the earlier time for the under coupling condition (Figure 4(a), profile 1) showed almost no diffraction; the diffraction effect increased from intermediate to later time profiles (Figure 4(a), profiles 2 and 3). This is reasonable because the profiles at the later time traveled effectively longer propagation distances. The later time profile for the under coupling condition (Figure 4(a), profile 3 ) was followed by the intermediate time profile for critical coupling condition (Figure 4(b), profile 2). The image further developed from intermediate to later time profiles with increasing diffraction effect (Figure 4(b), profiles 2 and 3). Finally, the later time profile for the critical coupling condition (Figure 4(b), profile 3) was followed by the intermediate time profile for the over coupling condition (Figure 4(c), profile 2). This time development is reasonable because the diffraction effect from the multiple-reflected

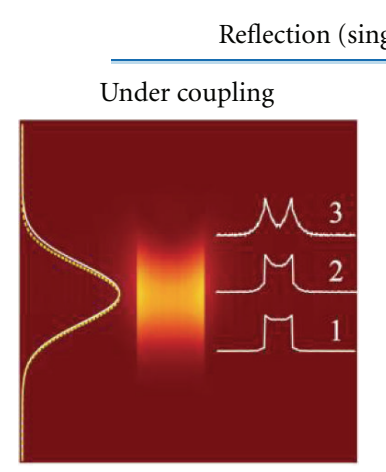

(a)

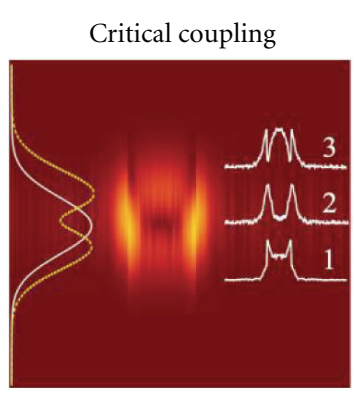

(b)

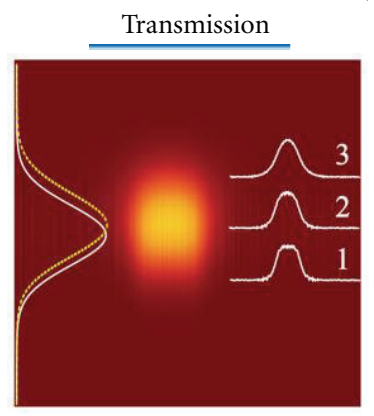

(d)

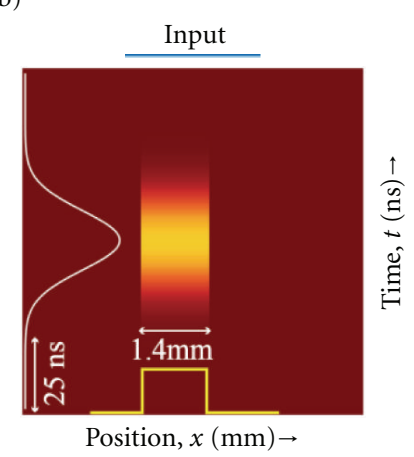

(e)

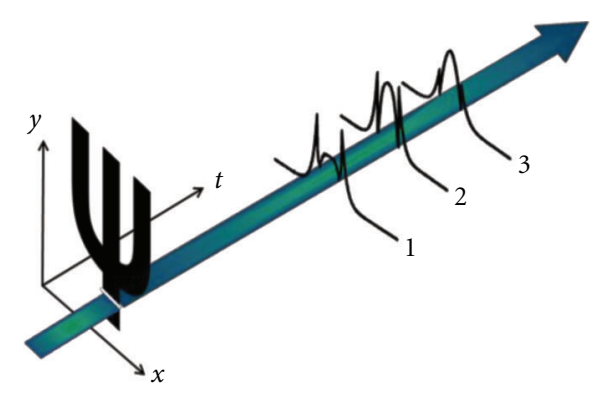

(f)

Figure 4: (a)-(d) Numerical simulation of the reflected and transmitted images in the space-time $(x-t)$ domain for a single resonator. (a)-(c) Reflected images from the single resonator for under, critical, and over coupling conditions, respectively. (d) Transmitted image from the single resonator. (e) Input image of width $1.4 \mathrm{~mm}$ (the horizontal axis) encoded on the temporal $25 \mathrm{~ns}$ Gaussian pulse (the vertical axis). (f) Illustration of the crosssection of the $2 \mathrm{D}$ image that was considered for time evolution analysis. The earlier, intermediate, and later time profiles are indicated by the notations 1, 2, and 3, respectively, in Figures 4(a)$4(\mathrm{~d})$. 
Size dependence (coupled resonator)

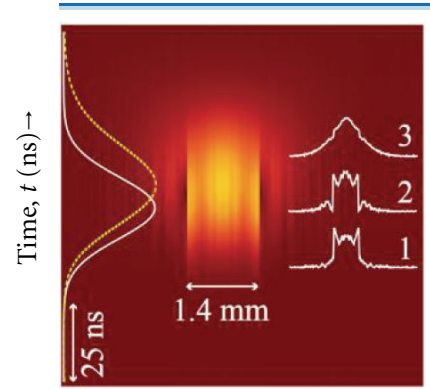

(a)

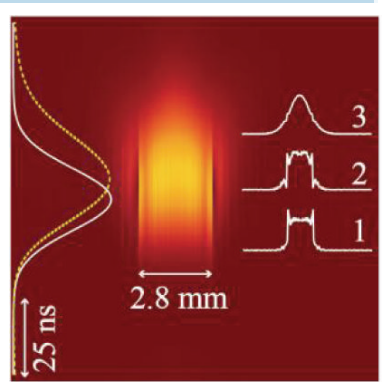

(b)
Position, $x(\mathrm{~mm}) \rightarrow$

Figure 5: (a) and (b) Reflected images from the coupled resonator for different image sizes. The sizes of the images used in (a) and (b) are $1.4 \mathrm{~mm}$ and $2.8 \mathrm{~mm}$, respectively, the full widths of the Fourier images are $\delta k=5.5 \mathrm{~mm}^{-1}$ and $\delta k=2.7 \mathrm{~mm}^{-1}$, respectively, and the spatial bandwidth of the cavity is $\Delta k=9.0 \mathrm{~mm}^{-1}$. The notations 1, 2, and 3 correspond to the earlier, intermediate, and later time profiles, respectively, in both figures. In both cases, the cavity parameters $\left(R_{1}=0.5, R_{2}=0.9, R_{3}=0.99, L_{1}=5 \mathrm{~cm}\right.$, $L_{2}=10 \mathrm{~cm}$ ) are the same as those used for the simulations shown in Figures 3(f) and 3(g).

components increased as the observation time or coupling strength increased.

A similar Fourier analysis approach was applied to the coupled image resonator. Figures 5(a) and 5(b) are simulations of the reflected cross-sectional images of different sizes from the coupled resonator. In Figures 5(a) and 5(b), images with a cross-section of $1.4 \mathrm{~mm}$ and $2.8 \mathrm{~mm}$ were used as inputs, respectively. The cavity parameters were the same as those shown in Figures 3(f) and 3(g). The full widths of the Fourier images were $\delta k=5.5 \mathrm{~mm}^{-1}$ and $\delta k=2.7 \mathrm{~mm}^{-1}$, respectively, and the $k$-space bandwidth of the cavity was $\Delta k=9.0 \mathrm{~mm}^{-1}$. The frequency bandwidths of the image and cavity were $\delta v=17.6 \mathrm{MHz}$ and $\Delta \nu=$ $80.6 \mathrm{MHz}$ in both cases. The image that propagated through the coupled resonator had aspects of both under and over coupling conditions: the edge-enhancement effect at the earlier time due to the under coupling condition in the first cavity and the delayed time profile due to the over coupling condition in the second cavity. In Figure 5(a), we observed a striped structure. When the image size was reduced, the higher Fourier components of the image overlapped the higher cavity resonance modes, which led to the striped effect.

\section{Experimental Demonstration}

The present Fourier analysis not only reproduces the reported experimental observations $[15,16]$ but also predicts new aspects of the time evolution of image propagation through the cavity; the propagated image profiles are time dependent. To demonstrate the new aspect of the time evolution, we performed time- and space-resolved image propagation experiments. The experimental setup is illustrated in Figure 6(a). While various configurations are possible in the image resonators, we studied a particular case using an over coupled single resonator system corresponding to the simulation of Figure 4(c), because the time evolution effect appeared clearly in this system. The light source for the experiment was a continuous wave $\mathrm{Nd}^{+3}$ :yttrium aluminum garnet (Nd:YAG) laser. The Nd:YAG laser, operated with a linewidth of $1 \mathrm{kHz}$, produced second-harmonic $532 \mathrm{~nm}$ light. The laser beam was expanded using two lenses (not shown in Figure 6(a)) to a diameter of $3 \mathrm{~cm}$. An image mask with the shape of the symbol " $\psi$ " was inserted into the collimated beam. The parameters used in this experiment were $R_{1}=0.7, R_{2}=0.9$, and $L=30 \mathrm{~cm}$, which corresponded to $\Delta k=5.9 \mathrm{~mm}^{-1}$. An image mask of width $1.6 \mathrm{~mm}\left(\delta k=4.7 \mathrm{~mm}^{-1}\right)$ was used for this experiment, and the injected temporal pulse width was $40 \mathrm{~ns}(\delta \nu=$ 11.0 MHz). While in the previous experiments $[15,16]$, the delay time was examined at a specific spatial point, here, we used a streak camera with a time resolution of $10 \mathrm{ps}$. The streak camera was a suitable device for the present experiment because it could analyze both the space and time domains simultaneously. The variable slit at the streak camera entrance was used to select the vertical height of the image.

Figures 6(b) (A) and 6(b) (B) show the experimental observation of the reflected off-resonance and on-resonance images, respectively. The experimental result shown in Figure 6(b) (B) corresponds to the simulation in Figure 4(c). In Figure 6(b) (C), the solid blue and red profiles are the results observed from the edge region and central region of the on-resonance image, respectively. The blackdashed line is the temporal profile obtained from the offresonance image of Figure 6(b) (A). Profiles (1), (2), and (3) are the cross-sectional profiles of the earlier, intermediate, and later times of the image in Figure 6(b) (B), respectively. The observed propagated spatial patterns were time dependent. It was also noticeable from Figure 6(b) (B) that the central region of the image was more delayed (upshifted), compared with the edge and off-resonance image. This result showed good agreement with the discussion for the over coupling condition (Figure 4(c)) and experimentally verified the analysis based on the Fourier approach. The off-resonance image (Figure 6(b) (A)) showed some fringe structures. This structure may be attributed to free space propagation from the image mask to the resonator and from the resonator to the streak camera.

\section{Conclusion}

In summary, we analyzed the propagation of $2 \mathrm{D}$ images encoded on temporal pulses through single and coupled resonators in Fourier space, using amplitude and phase transfer functions. The resonators were capable of propagating images, either delayed or advanced, as long as the spatial and temporal Fourier components of the image were within the bandwidth of the resonator. We also studied the observation time and coupling strength-dependent spatial image profiles. It has proven difficult to this point to increase 


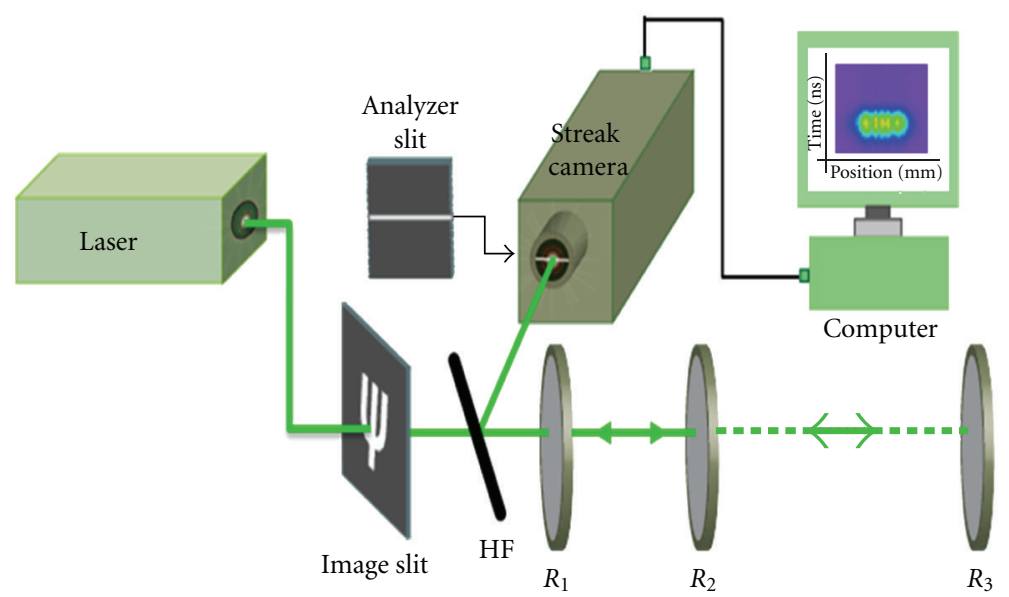

(a)

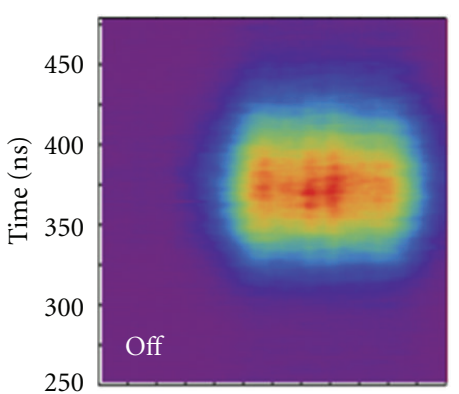

(A)

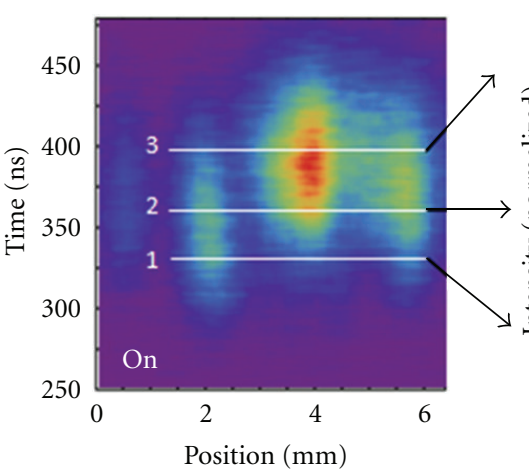

(B)

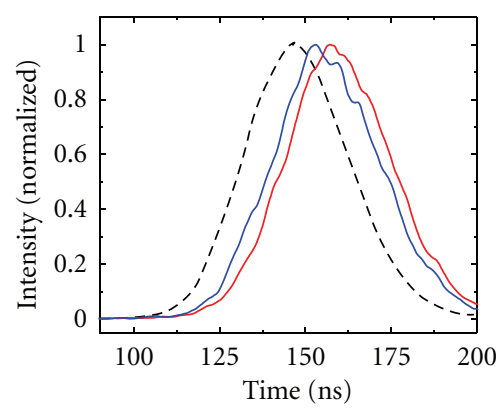

(C)

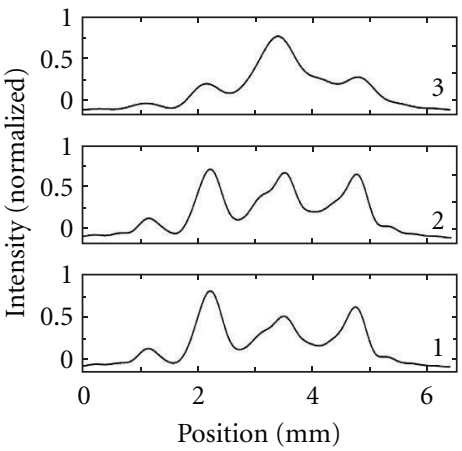

(D)

(b)

FIGURE 6: (a) Experimental setup for time- and space-resolved image propagation through the image resonator. Light pulses of 40 ns duration passed through an image mask and an image resonator system. The reflected images were sent to the streak camera using a half mirror (HF) to analyze time- and space-resolved reflected images. (b) (A) and (b) (B) show the reflected image for the off-resonance and on-resonance conditions, respectively. (b) (C) is the experimental observation of the temporal profiles of the reflected image. The dashed black curve is at the center of the off-resonance image; the solid blue and red curves are at the edge region and center region of the on-resonance image, respectively. (b) (D) is the cross-sectional profile of image (b) (B). The notations 1, 2, and 3 indicate the earlier, intermediate, and later times of the image, respectively.

the $k$-space bandwidth for realistic image applications. We can consider several possible solutions for this dilemma. One such possibility would be the use of a transverse degenerate paraxial stable Fabry-Perot cavity, consisting of confocal mirrors [18]. Previous studies have shown that such a cavity can transmit the self-transformed portion of the image [17]. Another possibility would be to introduce image guide photonic structures, such as those used in endoscopes or fiber image plates. If we can introduce a cavity structure into these image guide photonic structures, then this may improve the $k$-space bandwidth significantly, allowing the transmission of fine images. It should be noted that, in this case, the steep dispersion of the cavities could also yield fast and slow images, as discussed in this paper. 


\section{References}

[1] K. Y. Song, M. G. Herráez, and L. Thévenaz, "Gain-assisted pulse advancement using single and double Brillouin gain peaks in optical fibers," Optics Express, vol. 13, no. 24, pp. 9758-9765, 2005.

[2] M. A. I. Talukder, Y. Amagishi, and M. Tomita, "Superluminal to subluminal transition in the pulse propagation in a resonantly absorbing medium," Physical Review Letters, vol. 86, no. 16, pp. 3546-3549, 2001.

[3] L. J. Wang, A. Kuzmich, and A. Dogariu, "Gain-assisted superluminal light propagation,” Nature, vol. 406, no. 6793, pp. 277-279, 2000.

[4] L. V. Hau, S. E. Harris, Z. Dutton, and C. H. Behroozi, "Light speed reduction to 17 metres per second in an ultracold atomic gas," Nature, vol. 397, no. 6720, pp. 594-598, 1999.

[5] M. M. Kash, V. A. Sautenkov, A. S. Zibrov et al., "Non-linear magneto-optics and reduced group velocity of light in atomic vapor with slow ground state relaxation," Physical Review Letters, vol. 82, pp. 5229-5232, 1999.

[6] K. Totsuka and M. Tomita, "Slow and fast light in a microsphere-optical fiber system," Journal of the Optical Society of America B, vol. 23, no. 10, pp. 2194-2199, 2006.

[7] K. Totsuka, N. Kobayashi, and M. Tomita, "Slow light in coupled-resonator-induced transparency," Physical Review Letters, vol. 98, no. 21, Article ID 213904, 4 pages, 2007.

[8] K. Di, C. Xie, and J. Zhang, "Coupled-resonator-induced transparency with a squeezed vacuum," Physical Review Letters, vol. 106, Article ID 153602, 4 pages, 2011.

[9] C. Liu, Z. Dutton, C. H. Behroozi, and L. V. Hau, "Observation of coherent optical information storage in an atomic medium using halted light pulses," Nature, vol. 409, no. 6819, pp. 490493, 2001.

[10] R. Walsworth, S. Yelin, and M. Lukin, "The story behind 'stopped light"' Optics and Photonics News, vol. 13, no. 5, pp. 50-76, 2002.

[11] R. M. Camacho, C. J. Broadbent, I. A. Khan, and J. C. Howell, "All-optical delay of images using slow light," Physical Review Letters, vol. 98, no. 4, Article ID 043902, 4 pages, 2007.

[12] P. K. Vudyasetu, R. M. Camacho, and J. C. Howell, "Storage and retrieval of multimode transverse images in hot atomic rubidium vapor," Physical Review Letters, vol. 100, no. 12, Article ID 123903, 4 pages, 2008.

[13] M. Shuker, O. Firstenberg, R. Pugatch, A. Ron, and N. Davidson, "Storing images in warm atomic vapor," Physical Review Letters, vol. 100, no. 22, Article ID 223601, 4 pages, 2008.

[14] O. Firstenberg, M. Shuker, N. Davidson, and A. Ron, "Elimination of the diffraction of arbitrary images imprinted on slow light," Physical Review Letters, vol. 102, no. 4, Article ID 043601, 4 pages, 2009.

[15] M. Tomita, P. Sultana, A. Takami, and T. Matsumoto, "Advanced and delayed images through an image resonator," Optics Express, vol. 18, no. 12, pp. 12599-12605, 2010.

[16] P. Sultana, A. Takami, T. Matsumoto, and M. Tomita, "Delayed optical images through coupled-resonator-induced transparency," Optics Letters, vol. 35, no. 20, pp. 3414-3416, 2010.

[17] S. Gigan, L. Lopez, N. Treps, A. Maaetre, and C. Fabre, "Image transmission through a stable paraxial cavity," Physical Review A, vol. 72, no. 2, Article ID 023804, 10 pages, 2005.

[18] J. A. Arnaud, "Degenerate optical cavities," Applied Optics, vol. 8, no. 1, pp. 189-195, 1969.
[19] J. W. Goodman, Introduction to Fourier Optics, McGraw-Hill, New York, NY, USA, 1968. 

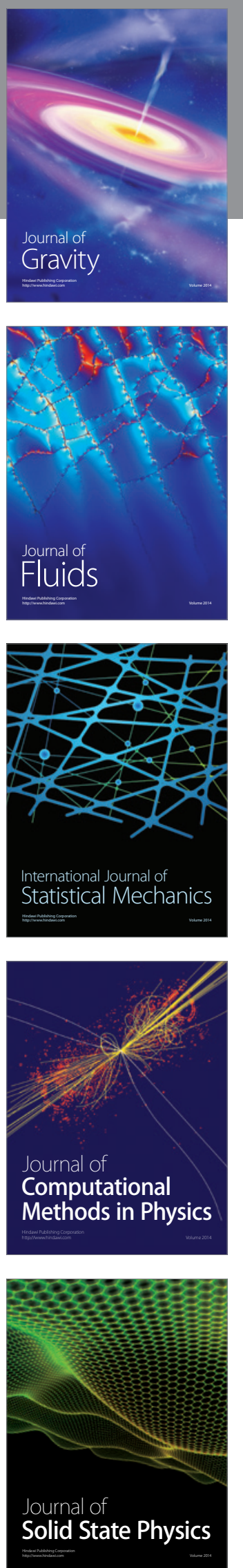

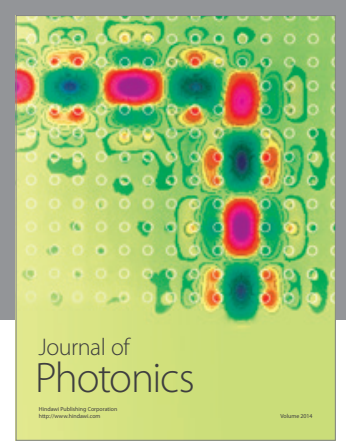

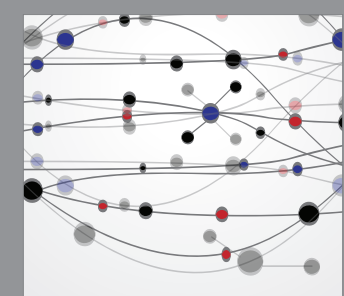

The Scientific World Journal
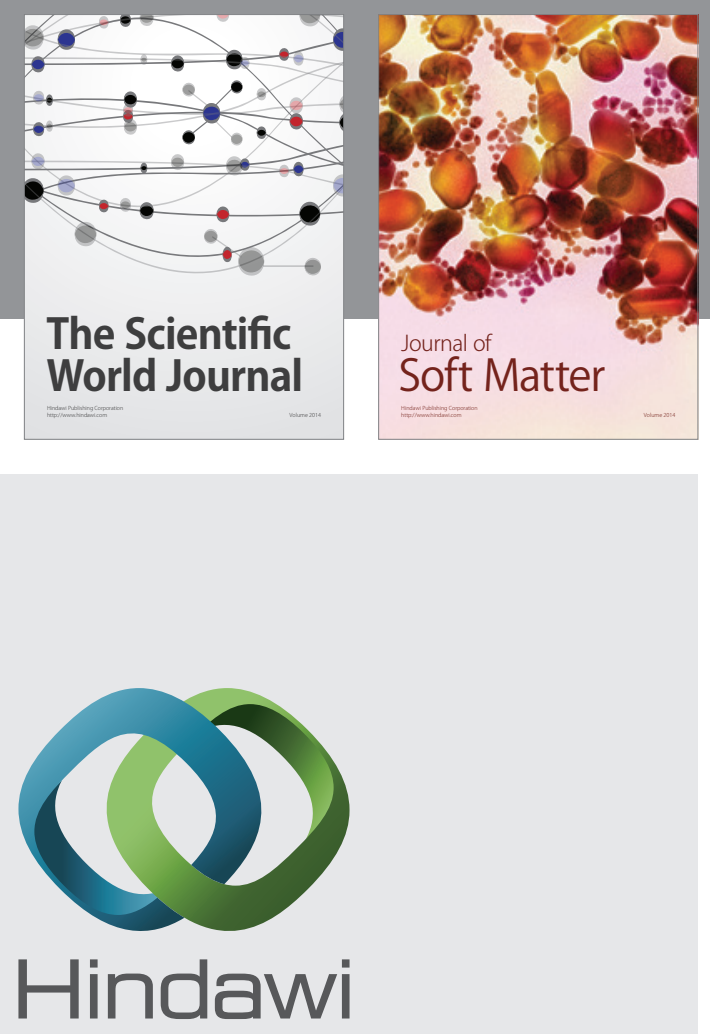

Submit your manuscripts at

http://www.hindawi.com
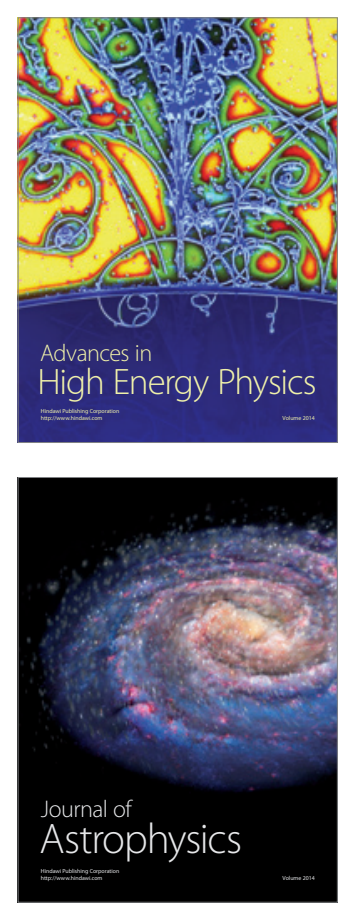
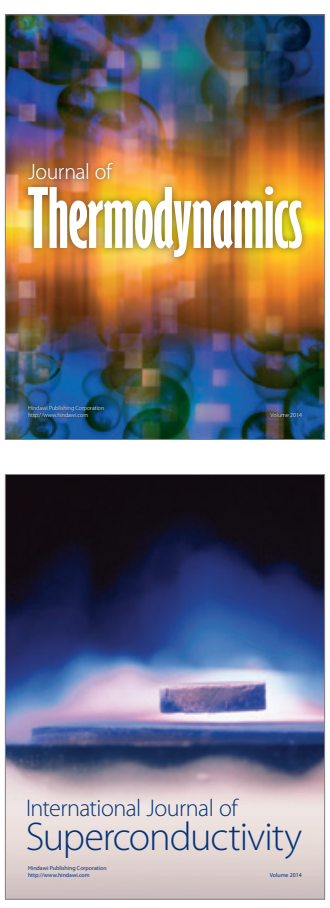
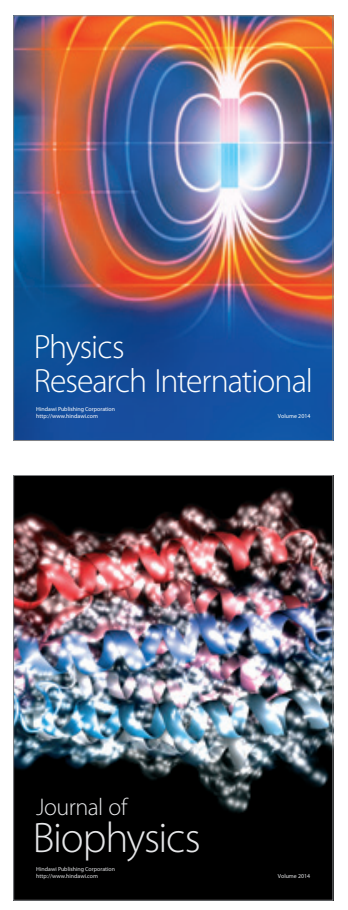
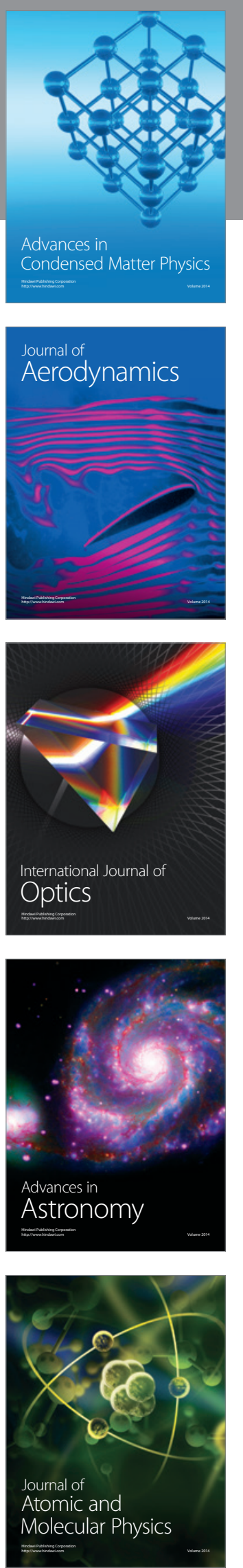